\title{
The endoscopic anatomy of the cochlear hook region and fustis: surgical implications
}

\author{
Anatomia endoscopica della "cochlear hook region" e del fustis: \\ importanza per la chirurgia
}

\author{
L. ANSCHUETZ ${ }^{12}$, M. ALICANDRI-CIUFELLI ${ }^{13}$, W. WIMMER ${ }^{24}$, M. BONALI ${ }^{1}$, M. CAVERSACCIO ${ }^{24}$, L. PRESUTTI \\ ${ }^{1}$ Department of Otolaryngology Head and Neck Surgery, University Hospital of Modena, Italy; ${ }^{2}$ Department \\ of Otolaryngology Head and Neck Surgery, Inselspital, University Hospital and University of Bern, Switzerland; \\ ${ }^{3}$ Neurosurgery Department, New Civil Hospital Sant'Agostino-Estense, Baggiovara (MO), Italy; ${ }^{4}$ Artificial Hearing \\ Research, ARTORG Center for Biomedical Engineering, University of Bern, Switzerland
}

\section{SUMMARY}

The cochlear hook region can be considered as the interface between the middle and inner ear. The identification of surgically-relevant endoscopic landmarks of this anatomical entity and assessment of their clinical value is still lacking in the literature. Procedures like cholesteatoma surgery and minimal invasive endoscopic approaches to the lateral skull base may particularly benefit from these considerations. We hypothesize that the spatial orientation of anatomical landmarks in the cochlear hook can be expressed in angles and are reproducibly identifiable by transcanal otoendoscopy. Therefore, endoscopic dissection of the cochlear hook region was performed in 32 temporal bone specimens. Topographic anatomy was documented and analysed. We performed computed tomography of 28 specimens to assess the region in three-dimensional reconstructions. The mean angle between the round window and the basal scala tympani was assessed $25.9^{\circ}$ in endoscopic and $28.2^{\circ}$ in three-dimensionally reconstructed models. The fustis was recognised as a reliable landmark for the basal turn. A mean angle of $155.4^{\circ}$ to the basal scala tympani was assessed. A slight bulging without obstruction of the basal turn was observed in 5 cases. The utility of the revealed anatomical details was assessed in minimal invasive endoscopic lateral skull base approaches. In conclusion, we described the angles between anatomical landmarks of the cochlear hook region. Moreover, the angle as recorded through an endoscope was found to be reliable compared to three-dimensional reconstructions from computed tomography.

KEY WORDS: Cochlear hook region • Endoscopic ear surgery • Cochlear implant $\bullet$ Lateral skull base surgery $\bullet$ Fustis $\bullet$ Cholesteatoma $\bullet$ Vestibular schwannoma

\section{RIASSUNTO}

La “cochlear hook region" (CHR) può essere considerata come una interfaccia tra l'orecchio medio e l'orecchio interno. L'identificazione endoscopica dei reperi chirurgici della CHR e la valutazione della loro rilevanza clinica non sono ancora state descritte in letteratura. Specialmente la chirurgia del colesteatoma e gli approcci mini-invasivi al basicranio laterale possono beneficiare di queste considerazioni. Ipotizziamo in questo lavoro che l'orientamento spaziale dei reperi chirurgici nella CHR possono essere espressi in forma di angoli e risultano essere riproducibili in approcci otoendoscopici. 32 dissezioni di ossa temporali sono state condotte in questo lavoro. L'anatomia topografica è stata documentata e analizzata. Una TC è stata eseguita in 28 temporali per valutare l'anatomia attraverso ricostruzioni 3D. L'angolo medio tra la finestra rotonda e il giro basale della chiocciola (scala tympani) è risultato essere di $25,9^{\circ}$ in endoscopia, e di $28,2^{\circ}$ nelle ricostruzioni $3 D$. Il fustis ha rappresentato un repere affidabile per il giro basale della chiocciola, con un angolo medio di $155,4^{\circ}$ con il giro basale della scala tympani. Una lieve prominenza senza ostruzione del giro basale della chiocciola è stata osservata in 5 casi. L'utilità di questi rilievi anatomici è stata confermata negli approcci mini-invasivi al basicranio laterale. In conclusione gli angoli tra i reperi anatomici della CHR così come descritti attraverso gli approcci endoscopici forniscono un orientamento utile per procedure come impianti cocleari, approcci mini-invasivi all'apice petroso o accessi transpromontoriali al condotto uditivo interno.

PAROLE CHIAVE: Cochlear hook region $\bullet$ Chirurgia endoscopica dell'orecchio $\bullet$ Impianto cochleare $\bullet$ Chirurgia endoscopica basicranio laterale $\bullet$ Fustis $\bullet$ Cholesteatoma $\bullet$ Schwannoma vestibulare

\section{Introduction}

The cochlear hook region represents an important interface between the middle ear and lateral skull base including the inner ear. It represents the most basal part of the cochlea and contains structures such as the round window mem- brane (RWM), the stapes footplate, the vestibule connected to the scala vestibuli (SV), the ductus reuniens and the scala tympani (ST) ${ }^{12}$. The position of the basal turn (BT) and its relationships to middle ear structures are of special surgical interest, especially in cochlear implant (CI) surgery ${ }^{3}$. With the emergence of minimally-invasive, transcanal en- 
doscopic techniques in ear and lateral skull base surgery, there is a growing interest in describing the topographical anatomy of the cochlear hook region from an endoscopic perspective. It is particularly important in the infracochlear approach ${ }^{4}$, where access to the inferior petrous apex is performed between the BT, jugular bulb (JB) and internal carotid artery (ICA). The transcanal transpromontorial approach to the internal auditory canal (IAC) requires exposure and dissection of the hook region before accessing the IAC. The anatomy of the cochlear hook region and the vestibule allows prediction of the position of the labyrinthine portion of the facial nerve (FN). This is important in order to safely remove the pathology from the IAC whilst preserving the $\mathrm{FN}^{5-7}$.

However, an endoscopic description of the cochlear hook region is still lacking in the literature. We hypothesise that the angles measured between anatomical landmarks are reproducibly identifiable using the endoscope when compared to high resolution computed tomography (HRCT). Therefore, we compared endoscopically assessed angles to threedimensional reconstructions of HRCT scans of the temporal bone. This work aims to provide the surgeon with anatomical details, which are directly applicable during surgery.

\section{Materials and methods}

\section{Endoscopic dissection}

The local institutional review board granted approval to perform the present study (KEK-BE 2016-00887). We conducted cadaveric dissection on human whole head and temporal bone specimens. We used a $0^{\circ}, 3 \mathrm{~mm}$ diameter and $14 \mathrm{~cm}$ length endoscope connected to a high-resolution camera system and monitor (Karl Storz, Tuttlingen, Germany).

The endoscopic access to the middle ear was obtained through the external auditory canal (EAC) performing a routine tympanomeatal flap. First, we performed a thorough exploration of the middle ear and documentation of the round window niche. To this end, we identified the subiculum, finiculus, subcochlear canaliculus, anterior and posterior pillars, tegmen and RWM. The fustis, a small bony plate, was identified on the floor of the subtympanic sinus running from the styloid prominence to the round window niche as shown in Figure $1^{89}$.

As the next step, we uncovered the cochlear hook region. The promontory bone was gently removed using small diamond burrs to expose the RMW, vestibule, SV ST, spiral lamina and spiral ligament. Figure 2 gives an illustrative overview on the endoscopic landmarks of the cochlear hook region.

To answer the study questions, we assessed following anatomical relationships:

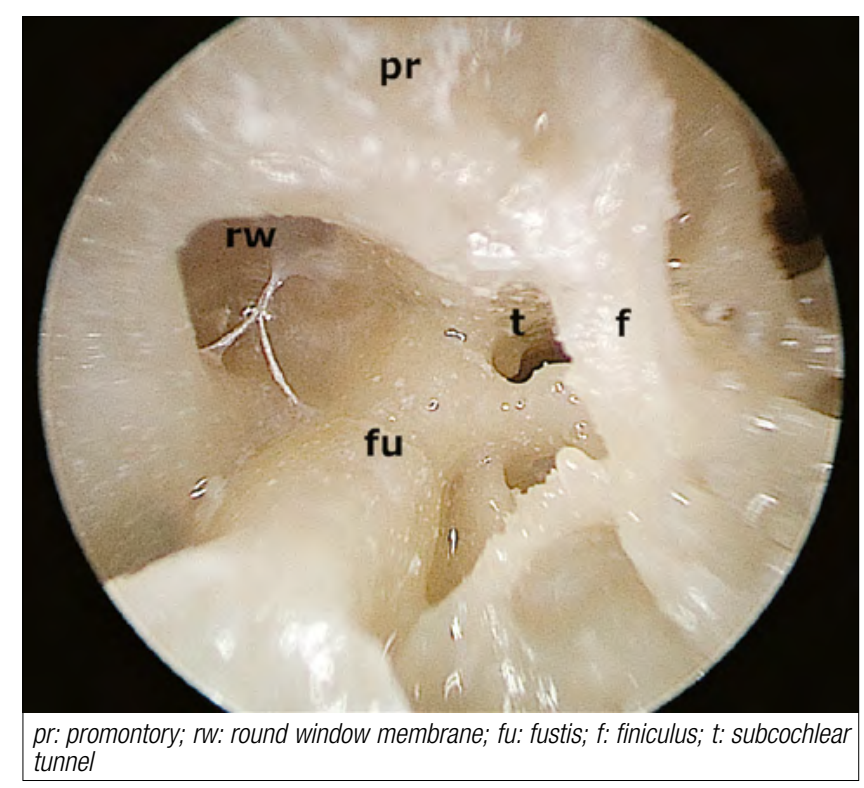

Fig. 1. Round window niche. Right ear: Details of important middle ear landmarks for the surgical treatment of the cochlear hook region. Note the direction of the fustis pointing towards the basal turn and representing the superior limit of the subcochlear tunnel.

- The fenestro-basal angle (FBA): the angle between a line approximated through the RWM center and the center of the basal ST was defined and measured from endoscopic photography.

- Morphology of the fustis: the orientation of the fustis and its configuration in relation to the RWM and the BT were assessed. Based on photographs taken with the endoscope, we measured the angle between the fustis and the BT.

- Inside the $S T$, an eventual bulging of the lateral cochlear wall into the hook region was assessed.

\section{Three-dimensional assessment of the fenestro-basal angle}

To investigate the suitability of an endoscopic view to determine the above-mentioned angles, 28 sides were imaged with HRCT scans (SOMATOM Definition Edge, Siemens, Erlangen, Germany) with a voxel size of $0.156 \times 0.156 \times 0.2 \mathrm{~mm}^{3}$ before dissection. In the images, the cochlea, round window and promontorial bone were segmented, and three-dimensional surface models were created. A semi-automatic algorithm based on the selection of anatomical landmarks including the centre of the RWM ${ }^{10}$ was used to approximate the centerline of the scala tympani in the cochlear basal turn. The tangent of the ST centre line was computed at the RW centre. The orientation of the RW membrane (which has a curved shape) was approximated by fitting a plane through the coordinates of the 20 closest RW membrane vertices in the vicinity of the RW center. The FBA was assessed as the angle between the fitted round window plane and tangential vector of the ST centerline (Fig. 3). 


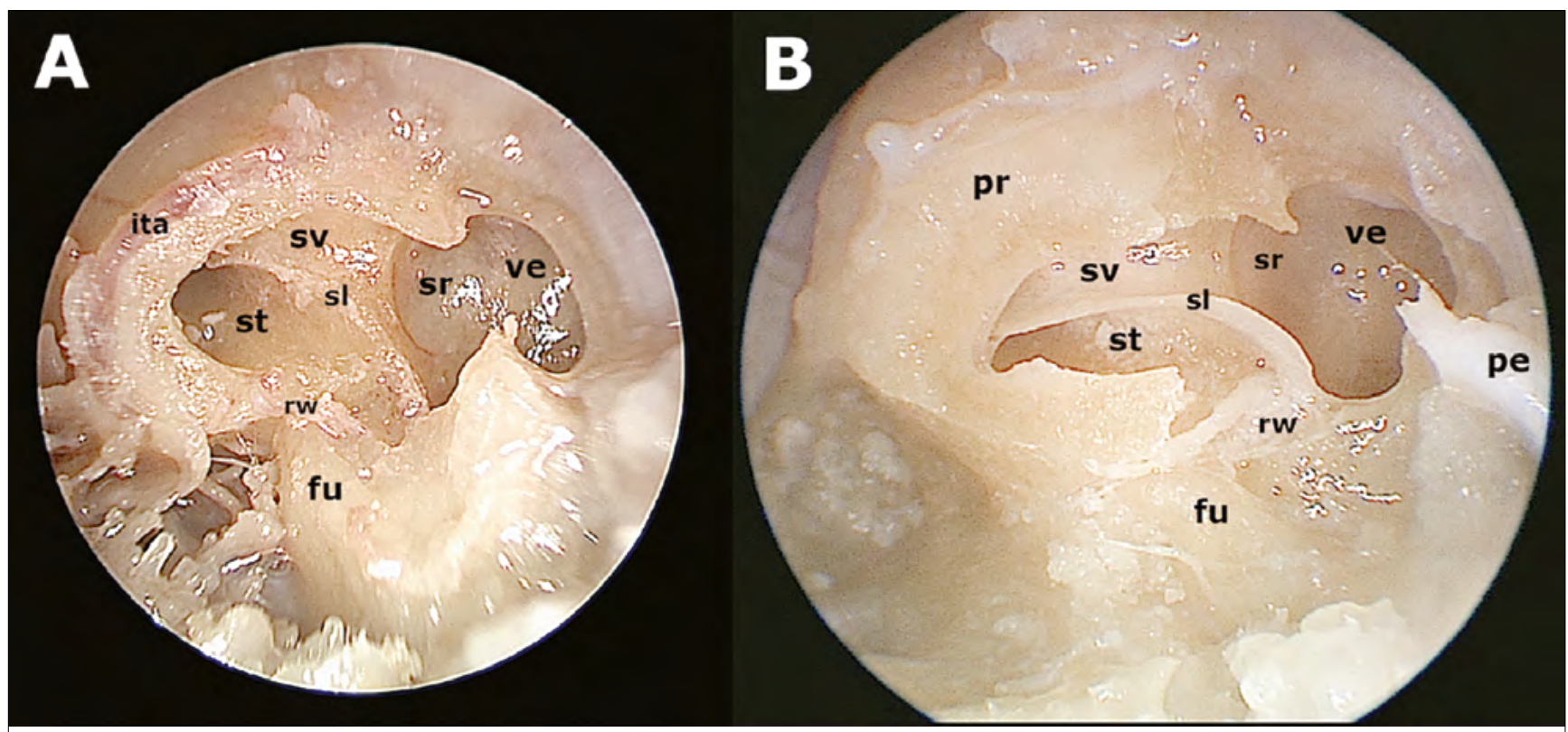

ita: inferior tympanic artery; sv: scala vestibuli; st: scala tympani; sl: spiral lamina; sr: spherical recess; rw: round window membrane; ve: vestibule; fu: fustis; pe: pyramidal eminence; pr: promontory.

Fig. 2. Hook region anatomy. Left ear: The endoscopic anatomy of the cochlear hook region in overview (A) and in detail; (B) after removal of the promontory bone and the stapes.

\section{Statistical analysis}

A two-tailed Wilcoxon matched-pairs signed rank test was performed to evaluate the association between the angles measured in endoscopic dissection with the angles measured in the three-dimensional model. All analyses were conducted using GraphPad Prism $7^{\circledR}$. Alpha for statistical significance was set at 0.05 .

\section{Results}

We analysed a total of 32 sides. The landmarks were well identifiable in all cases using the transcanal endoscopic approach. Figure 1 gives an overview over the important endoscopic landmarks of the round window niche and Figure 2 on the cochlear hook region.

The mean FBA was assessed endoscopically $25.9^{\circ}$ $\left( \pm 5.6^{\circ}\right)$ and radiologically $28.2^{\circ}\left( \pm 10.9^{\circ}\right)$. The main difference of $2.3^{\circ}$ between the endoscopic and radiologic assessment was not statistically significant $(\mathrm{p}=0.23$ ).

A small bulging of bone into the basal ST at the level of the anterior pillar of the RWM was identified in 5 cases $(15.6 \%)$. However, we observed only minor narrowing of the ST width.

The fustis bone was identifiable in all specimens and was pneumatised inferiorly in 14 cases $(43.8 \%)$. Concerning

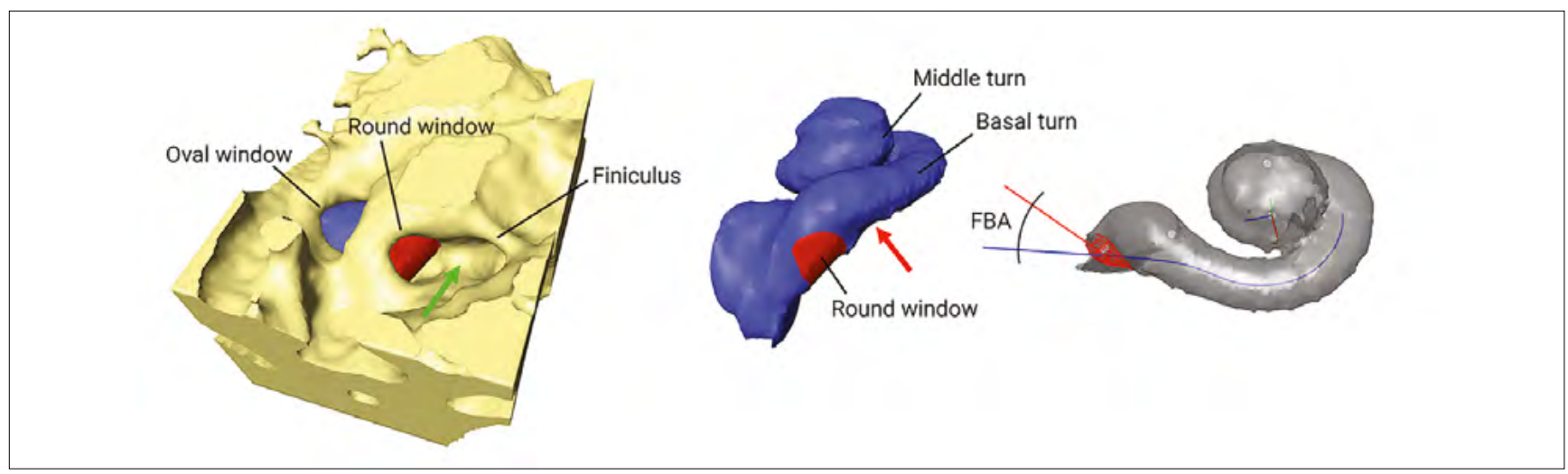

Fig. 3. Three-dimensional model. Three-dimensional model of a right cochlear hook region and the finestro-basal angle (FBA) measured as a normal vector to the round window membrane and the center of the basal scala tympani. The green arrows indicate the fustis, and the red arrow indicates a bony bulging into the scala tympani. 
the direction of the fustis, we observed three kinds of configurations:

1. Horizontal: the fustis runs from the styloid prominence posteriorly to the anterior part of the RWM, directly pointing to the basal ST ( $\mathrm{n}=14 ; 43.8 \%)$.

2. Inferior: the fustis points inferior to the basal ST just anterior to the RWM $(n=12 ; 37.5 \%)$.

3. Fused: the fustis is fused to the bone of the area concamerata $(n=6 ; 18.7 \%)$.

The mean angle between the tangential vector of the fustis and the centre line of the ST was measured $155.4^{\circ}\left( \pm 16.2^{\circ}\right)$.

\section{Discussion}

This study describes refined surgical landmarks and relationships of the cochlear hook region from an endoscopic view. Previous descriptions of the round window niche and the cochlear hook anatomy showed abundant inter-individual anatomical variability. Su et al. dissected 549 temporal bones, measuring the transverse diameter of the round window membrane with a mean of $1.65 \mathrm{~mm}$ (range: 0.96-2.28) and the depth of the round window niche had a mean of $1.34 \mathrm{~mm}$ (range: 0.69-2.28), thus representing a large variability especially in terms of size of the analysed structures ${ }^{11}$. Tóth et al. investigated 783 temporal bones endoscopically and described the anatomical variability of the round window niche ${ }^{12}$. In the same line, abundant anatomical variability was recently published on a series of 23 temporal bones. The authors provide interesting measures such as the distance between the center of the oval window $(\mathrm{OW})$ and the RW rim $(1.50 \mathrm{~mm}$, range: 1.11-2.24) or the distance between the anterior rim of the OW and the anterior rim of the RW $(3.26 \mathrm{~mm}$, range: $2.31-3.94){ }^{1}$. Another interesting publication observed $7.5 \%$ cases with basal turn constrictions ${ }^{13}$. Actual$1 y$, in our dissection we also observed $15.6 \%$ of cases with minimal bulging of the hook region into the basal turn. However, we did not observe any obstruction of the ST. In contrast to previous descriptions of the cochlear hook regions, where topographical relationships were expressed by measuring distances between landmarks, we aimed to add the angulation of the structures to the topographical description. Due to the panoramic views offered by the endoscope, the surgeon is able to understand the angles between middle ear landmarks (RWM or fustis) and the inner ear (BT). Knowledge about these relationships allows the surgeon to draw conclusions about the spatial orientation of the cochlear hook region. Therefore, the FBA was defined as the angle between the RWM and the basal ST. When comparing these values to a three-dimensional reconstruction of the cochlea from HRCT scans, a similar angle of $28^{\circ}$ was observed, which indicates good reliability of endoscopically perceived angles. The same considerations apply for the angle between the fustis and the basal ST.

These topographical descriptions are particularly interesting in the context of minimally-invasive endoscopic skull base surgery. The first example to mention is the subcochlear approach ${ }^{4}$. This transcanal route is used to treat pathologies of the inferior petrous apex (e.g. cholesteatoma, cholesterol granuloma) conserving the ossicular chain and the cochlea, and therefore hearing. The cochlear hook region represents in this case the superior limit of the approach that is important for surgical access (14). The second application of the FBA in endoscopic lateral skull base surgery is the exclusive endoscopic transcanal transpromontorial approach to the IAC. This approach is used to remove small vestibular schwannomas in patients with unserviceable hearing ${ }^{5}$. The fustis and the BT are important landmarks as they indicate the inferior and posterior limit of the IAC fundus.

Marchioni et al. presented in 2015 an endoscopic classification of the round window niche and highlighted its anatomical aspects in 65 surgical cases ${ }^{8}$. In that study, the authors emphasised the utility of the endoscope to assess the round window niche. We could reproduce these results in all specimens. In 2016 Marchioni et al. described the anatomy of the fustis bone in greater detail ${ }^{15}$. They proposed a classification for the fustis as type A: pointing to the RWM and type $\mathrm{B}$ pointing anterior and inferior to the RWM in direction of the BT. This classification is equivalent to our investigations (type $\mathrm{A}=$ horizontal and type $\mathrm{B}=$ inferior). However, according to our observations, we suggest to add a third type of fustis configuration to the classification: namely, the fusion of the fustis to the area concamerata (type $\mathrm{C}=$ fused, compare Fig. 2A). Another interesting observation was the pneumatisation of the fustis bone in $44 \%$ of cases. This suggests that a cholesteatoma could spread inside and below the fustis bone. In these cases, an infracochlear approach should be adopted to completely remove the pathology. It would be interesting to know whether the fustis pneumatisation is related to the pneumatisation of the subtympanic sinus, which would have additional considerations in cholesteatoma surgery ${ }^{15}$.

In our experience, the knowledge of the FBA also facilitates correct positioning of the $\mathrm{CI}$ array into the ST. This consideration may be the key clinical utility of the present investigation, as we would expect less injury to the basal spiral lamina and spiral ligament when inserting the array in a smooth way by respecting the natural direction of the ST. Steeper insertion angles may result in perforation of the spiral lamina and insertion of the array into the SV ${ }^{1}$. Lo et al. demonstrated in a guinea pig model the importance of the cochlear hook 
region in minimising trauma during CI. They found either the RW or the cochleostomy approach to be suitable to avoid insertion trauma ${ }^{17}$. This consideration gains clinical importance if the visibility of the round window niche through a facial recess approach is limited ${ }^{18} 19$. Thus, knowledge of the FBA and direction of the fustis may serve as reliable landmarks for successful array implantation.

\section{Conclusions}

The fenestro-basal angle was $26^{\circ}$ and the angle between the fustis and the basal ST was $155^{\circ}$. These measurements represent reliably identifiable topographical relationships during the endoscopic approach to the lateral skull base.

\section{Acknowledgements}

The authors wish to thank the Institute of Anatomy, University of Bern, Switzerland and especially Mrs. Nane Boemke for the provided anatomical specimens.

\section{Conflict of interest statement}

None declared.

\section{References}

1 Atturo F, Barbara M, Rask-Andersen H. On the anatomy of the 'hook' region of the human cochlea and how it relates to cochlear implantation. Audiol Neurootol 2014;19:378-85. https://doi.org/10.1159/000365585.

2 Li PM, Wang H, Northrop C, et al. Anatomy of the round window and hook region of the cochlea with implications for cochlear implantation and other endocochlear surgical procedures. Otol Neurotol 2007;28:641-8. https://doi.org/10.1097/mao.0b013e3180577949.

3 Stidham KR, Roberson JB Jr. Cochlear hook anatomy: evaluation of the spatial relationship of the basal cochlear duct to middle ear landmarks. Acta Otolaryngol 1999;119:773-7. https://doi. org/10.1080/00016489950180414.

4 Marchioni D, Alicandri-Ciufelli M, Rubini A, et al. Endoscopic transcanal corridors to the lateral skull base: initial experiences. Laryngoscope 2015;125(Suppl 5):S1-13. https://doi.org/10.1002/lary.25203.

5 Marchioni D, Alicandri-Ciufelli M, Rubini A, et al. Exclusive endoscopic transcanal transpromontorial approach: a new perspective for internal auditory canal vestibular schwannoma treatment. J Neurosurg 2016;11:1-8. https://doi.org/10.3171/2015.11.JNS15952.
6 Presutti L, Bonali M, Marchioni D, et al. Expanded transcanal transpromontorial approach to the internal auditory canal and cerebellopontine angle: a cadaveric study. Acta Otorhinolaryngol Ital 2017;37:224-30. https://doi.org/10.14639/0392-100X-1258.

7 Presutti L, Alicandri-Ciufelli M, Bonali M, et al. Expanded transcanal transpromontorial approach to the internal auditory canal: pilot clinical experience. Laryngoscope 2017;127:2608-14. https://doi. org/10.1002/lary.26559.

8 Marchioni D, Alicandri-Ciufelli M, Pothier DD, et al. The round window region and contiguous areas: endoscopic anatomy and surgical implications. Eur Arch Otorhinolaryngol 2015;272:1103-12. https:// doi.org/10.1007/s00405-014-2923-8.

9 Proctor B, Bollobas B, Niparko JK. Anatomy of the round window niche. Ann Otol Rhinol Laryngol 1986:95:444-6. https://doi. org/10.1177/000348948609500502.

10 Wimmer W, Venail F, Williamson T, et al. Semiautomatic cochleostomy target and insertion trajectory planning for minimally invasive cochlear implantation. Biomed Res Int 2014;2014:596498. https://doi. org/10.1155/2014/596498.

11 Su WY, Marion MS, Hinojosa R, et al. Anatomical measurements of the cochlear aqueduct, round window membrane, round window niche, and facial recess. Laryngoscope 1982;92:483-6. https://doi. org/10.1288/00005537-198205000-00003.

12 Tóth M, Alpár A, Patonay L, et al. Development and surgical anatomy of the round window niche. Ann Anat 2006;188:93-101. https://doi. org/10.1016/j.aanat.2005.09.006.

13 Singla A, Sahni D, Gupta AK, et al. Surgical anatomy of the basal turn of the human cochlea as pertaining to cochlear implantation. Otol Neurotol 2015;36:323-8. https://doi.org/10.1097/MAO.0000000000000371.

14 Anschuetz L, Presutti L, Schneider D, et al. Quantitative analysis of surgical freedom and area of exposure in minimal-invasive transcanal approaches to the lateral skull base. Otol Neurotol 2018;39:785-90.

15 Marchioni D, Soloperto D, Colleselli E, et al. Round window chamber and fustis: endoscopic anatomy and surgical implications. Surg Radiol Anat 2016;38:1013-9. https://doi.org/10.1007/s00276-016-1662-5.

16 Anschuetz L, Alicandri-Ciufelli M, Bonali M, et al. Novel surgical and radiologic classification of the subtympanic sinus: implications for endoscopic ear surgery. Otolaryngol Head Neck Surg 2018:194599818787180. https://doi.org/10.1177/0194599818787180.

17 Lo J, Sale P, Wijewickrema S, et al. Defining the hook region anatomy of the Guinea pig cochlea for modeling of inner ear surgery. Otol Neurotol 2017;38:e179-87. https://doi.org/10.1097/MAO.0000000000001446.

18 Alicandri-Ciufelli M, Fermi M, Bonali M, et al. Facial sinus endoscopic evaluation, radiologic assessment, and classification. Laryngoscope 2018;128:2397-402. https://doi.org/10.1002/lary.27135.

19 Williamson T, Gavaghan K, Gerber N, et al. Population statistics approach for safety assessment in robotic cochlear implantation. Otol Neurotol 2017;38:759-64. https://doi.org/10.1097/ MAO.0000000000001357.

Received: October 19, 2018 - Accepted: June 13, 2019

How to cite this article: Anschuetz L, Alicandri-Ciufelli M, Wimmer W, et al. The endoscopic anatomy of the cochlear hook region and fustis: surgical implications. Acta Otorhinolaryngol Ital 2019;39:353-357. https://doi.org/10.14639/0392-100X-2388

Address for correspondence: Lukas Anschuetz, Department of Otorhinolaryngology, Head \& Neck Surgery, University Hospital of Modena, Modena, Italy, via del Pozzo 71, 41100 Modena, Italy. Tel. +39 059 4222402. Fax +39 0594222454 . E-mail: anschuetz.lukas@ gmail. com

This is an open access article distributed in accordance with the Creative Commons Attribution Non Commercial (CC BY-NC 4.0) license, which permits others to distribute, remix, adapt, build upon this work non-commercially, and license their derivative works on different terms, provided the original work is properly cited, appropriate credit is given, any changes made indicated, and the use is non-commercial. See: http://creativecommons.org/licenses/by-nc/4.0/. 